\title{
Moving in the "right" direction: exposing subclinical right ventricular disease with exercise in patients with systemic sclerosis
}

\author{
Sarah Hale ${ }^{1} \cdot$ Benjamin H. Freed ${ }^{2}$
}

Published online: 17 May 2021

(C) The Author(s), under exclusive licence to Springer Nature B.V. 2021

\section{Commentary}

Exercise is a physiologic probe that effectively unmasks early stages of cardiovascular disease, potentially leading to earlier diagnosis and treatment for patients. While exercise is most commonly used to diagnose flow-limiting coronary artery disease, it may also aid in uncovering subclinical right ventricular dysfunction in patients at risk for pulmonary hypertension (PH). This type of tool might be uniquely helpful in patients with systemic sclerosis (SSc); many of whom have RV dysfunction with or without significant $\mathrm{PH}$. Unfortunately, there is a dearth of literature focused on early diagnosis and intervention in this understudied patient population with chronic, progressive disease.

In this issue of The International Journal of Cardiovascular Imaging, Mukherjee et al. address the importance of studying RV contractile reserve in SSc patients at risk for developing PH [1]. Using supine bicycle echocardiography, the authors measured RV functional parameters (including RV speckle tracking strain) at rest and at peak stress in order to calculate RV reserve. They found that RV free wall strain significantly improved at peak exercise with greater contractile response in the mid and apical segments compared to the basal segment. They further separated patients into two groups based on echocardiography-derived RV systolic pressure (RVSP) at rest to determine the effects of increased afterload on RV reserve. The authors discovered that SSc patients with RVSP $>35 \mathrm{mmHg}$ did not have any significant improvement in RV free wall strain compared to patients with RVSP $<35 \mathrm{mmHg}$, and there was evidence of

Benjamin H. Freed

benjamin.freed@northwestern.edu

1 Department of Medicine, Northwestern University Feinberg School of Medicine, Chicago, IL, USA

2 Department of Medicine, Northwestern University Feinberg School of Medicine, 676 North St. Clair St. Arkes Pavilion, Suite 600, Chicago, IL 60611, USA exertional RV dilation. The authors concluded that exercise provocation with RV strain allows for the early detection of subclinical RV disease which may, in turn, provide an opportunity to improve clinical outcomes.

There are a number of important findings from this study. First, there is growing evidence from these authors and others that the RV is particularly vulnerable to disease in patients with $\mathrm{SSc}$ regardless of $\mathrm{PH}[2,3]$. While most conventional 2D echocardiographic measures of RV function are unable to detect the initial deterioration associated with this disease, RV strain appears to be sensitive enough to detect both global and regional abnormalities not only at rest but with stress as well. This was nicely illustrated by the authors who showed that RV strain was the only measure to reveal significant differences in RV contractility with exercise between normal and elevated loading conditions. In addition, the regional changes in strain between rest and stress may offer new mechanistic insight into how the RV responds to the pressure and volume changes induced by exercise.

Second, the authors were able to show remarkable feasibility and reproducibility for a number of echocardiographic parameters at peak stress. This is critical if exercise for occult PH or subclinical RV dysfunction is ever going to become a standard in routine clinical practice. Acquiring data such as the tricuspid regurgitant velocity and RV free wall systolic strain is recommended by a recent EACVI/ASE document on the clinical use of stress echocardiography for $\mathrm{PH}$ and RV assessment [4], but poor acoustic windows during exercise can make this challenging. While others have shown success with obtaining the tricuspid regurgitant jet for measuring RVSP [5], this study is one of the few to show that successful acquisition of RV strain at peak stress is highly achievable.

Finally, one of the most significant messages from this study is the obvious need for more research in this space. Currently, there is no clear definition for what constitutes an abnormal pulmonary vasculature or $\mathrm{RV}$ response to exercise. 
Furthermore, there is no standardized method for how best to measure these responses, particularly in a non-invasive setting. Although RV strain appears to be an ideal surrogate for RV contractile reserve, there are no accepted reference values, making the interpretation of the results from this study challenging. What should RV strain be at peak exercise? What percent change in strain from rest is considered normal? Is maximal contraction truly reflected by an inability to increase strain? Only when these questions are answered can we start exploring the clinical relevance of abnormal RV reserve, as measured by RV strain, in SSc patients.

SSc is a chronic and debilitating disease with few treatment options. Since clinically evident cardiac disease has a poor prognosis in this patient population, the identification of subclinical disease and its response to therapy is of paramount importance. Mukherjee et al. should be congratulated for bringing attention to this rare disease and highlighting the utility of exercise provocation and speckle tracking strain in its management. There remains a significant amount of work to do before we incorporate these tools into clinical practice, but this study contributes greatly to the current literature and moves us closer to improving the clinical course of this disease.

Funding Not applicable.

Data and material availability Not applicable.

Code availability Not applicable.

\section{Declarations}

Conflict of interest The authors declare that they have no conflict of interest.

\section{References}

1. Mukherjee M, Mercurio V, Hsu S et al (2021) Assessment of right ventricular reserve utilizing exercise provocation in systemic sclerosis. Int J Cardiovasc Imaging. https://doi.org/10.1007/ s10554-021-02237-9

2. Tedford RJ, Mudd JO, Girgis RE et al (2013) Right ventricular dysfunction in systemic sclerosis-associated pulmonary arterial hypertension. Circ Heart Fail 6:953-963. https://doi.org/10.1161/ CIRCHEARTFAILURE.112.000008

3. Durmus E, Sunbul M, Tigen K et al (2015) Right ventricular and atrial functions in systemic sclerosis patients without pulmonary hypertension. Speckle-tracking echocardiographic study . Herz 40:709-715. https://doi.org/10.1007/s00059-014-4113-2

4. Lancellotti P, Pellikka PA, Budts W et al (2017) The clinical use of stress echocardiography in non-ischaemic heart disease: recommendations from the European Association of Cardiovascular Imaging and the American Society of Echocardiography. J Am Soc Echocardiogr 30:101-138. https://doi.org/10.1016/j.echo. 2016.10.016

5. Claessen G, La Gerche A, Voigt JU et al (2016) Accuracy of echocardiography to evaluate pulmonary vascular and RV function during exercise. JACC Cardiovasc Imaging 9:532-543. https:// doi.org/10.1016/j.jcmg.2015.06.018

Publisher's Note Springer Nature remains neutral with regard to jurisdictional claims in published maps and institutional affiliations. 